\title{
MIRIA: a webserver for statistical, visual and meta-analysis of RNA editing data in mammals
}

\author{
Xikang Feng ${ }^{1 \dagger}$, Zishuai Wang ${ }^{1 \dagger}$, Hechen $\mathrm{Li}^{1}$ and Shuai Cheng $\mathrm{Li}^{1,2^{*}}$ \\ From The International Conference on Intelligent Biology and Medicine (ICIBM) 2019 \\ Columbia, OH, USA. 09-11 June 2019
}

\begin{abstract}
Background: Adenosine-to-inosine RNA editing can markedly diversify the transcriptome, leading to a variety of critical molecular and biological processes in mammals. Over the past several years, researchers have developed several new pipelines and software packages to identify RNA editing sites with a focus on downstream statistical analysis and functional interpretation.

Results: Here, we developed a user-friendly public webserver named MIRIA that integrates statistics and visualization techniques to facilitate the comprehensive analysis of RNA editing sites data identified by the pipelines and software packages. MIRIA is unique in that provides several analytical functions, including RNA editing type statistics, genomic feature annotations, editing level statistics, genome-wide distribution of RNA editing sites, tissue-specific analysis and conservation analysis. We collected high-throughput RNA sequencing (RNA-seq) data from eight tissues across seven species as the experimental data for MIRIA and constructed an example result page.

Conclusion: MIRIA provides both visualization and analysis of mammal RNA editing data for experimental biologists who are interested in revealing the functions of RNA editing sites. MIRIA is freely available at https://mammal.

deepomics.org.
\end{abstract}

Keywords: RNA editing, Visualization, Analysis, Webserver, Mammal

\section{Background}

RNA editing is defined as a critical post-transcriptional regulatory RNA-processing event (excluding RNA splicing) that generates an RNA transcript with a primary nucleotide sequence different from its gene. In mammals, the most common form of RNA editing, A-to-I RNA editing, is catalysed by the ADAR family of enzymes (Adenosine Deaminase that Acts on RNA) [1, 2], and this process leads to an A-to-G reading of the cDNA molecule $[3,4]$. A-to-I RNA editing exists in the coding regions of many RNAs, including those encoding

\footnotetext{
*Correspondence: shuaicli@cityu.edu.hk

Xikang Feng and Zishuai Wang contributed to this work as first authors.

${ }^{1}$ Department of Computer Science, City University of Hong Kong, Kowloon, Hong Kong

${ }^{2}$ Department of Biomedical Engineering, City University of Hong Kong, Kowloon, Hong Kong
}

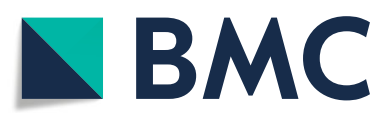

(c) The Author(s). 2019 Open Access This article is distributed under the terms of the Creative Commons Attribution 4.0 International License (http://creativecommons.org/licenses/by/4.0/), which permits unrestricted use, distribution, and

reproduction in any medium, provided you give appropriate credit to the original author(s) and the source, provide a link to the Creative Commons license, and indicate if changes were made. The Creative Commons Public Domain Dedication waiver (http://creativecommons.org/publicdomain/zero/1.0/) applies to the data made available in this article, unless otherwise stated.

glutamate receptor subunits [5-7], the G protein-coupled serotonin $2 \mathrm{C}$ receptor [8] and the anti-genome of the hepatitis delta virus $[9,10]$. The functional consequences of RNA editing in non-coding regions involve miRNA biogenesis [11], editing of miRNA seed regions [12] or target sequences within an mRNA [13] and nuclear retention [14]. Moreover, RNA editing has been shown to be associated with many diseases such as the autoimmune disorder Aicardi-Goutières syndrome [15], various viral infections [16] and different types of cancer [17].

Recently, increasing number of mammalian RNA editing databases have been published [18-22], there is a dire lack of online tools to perform mammalian RNA editing analysis. Therefore, we developed MIRIA (Mammalian RNA Editing Profiling and Interactive Analysis), a webserver which focuses on providing mammalian RNA editing statistics, genomic feature annotations, editing level calculations, 
genome-wide distributions of RNA editing sites, tissuespecific analyses and conservation analyses. Furthermore, we collected sequencing data of polyadenylated RNAs from eight organs (i.e., brain, heart, liver, spleen, lung, kidney, skeletal muscle, testis) across seven mammals (i.e., human, rhesus, rat, mouse, pig, cow, sheep) to test our webserver.

\section{Usage and implementation}

\section{Data uploading and filtering}

MIRIA is a new tool that was designed for RNA editing analysis in mammals. Users need to provide a compressed file (.zip) containing all RNA editing files from different mammals. The architecture of the zip file has two layers. The first layer contains all species folders (e.g., human, mouse, rat), and the second layer, which is inside each species folder, contains all RNA editing files of the species (e.g., tissue1.res, tissue2.res, tissue3.res). The format of the single RNA editing file (.res) is listed below (Table 1). By default, all RNA editing sites in the uploaded data are included to downstream analyses. Users have the option of filtering low-quality sites using the minimum supporting reads count cutoff and the minimum reads coverage cutoff on the uploading website interface. All sites that dissatisfy these criteria are excluded from downstream analyses.

\section{Percentage of all editing types}

Adenosine-to-inosine (A-to-I) editing is the most common form of editing in mammals. As such, the percentage of A-to-G editing, an indicator of A-to-I editing, is an important measurement to indirectly assess the detection accuracy of the RNA editing sites. Before calculating the percentage of A-to-G editing, we classified all editing sites into one of three categories, namely, those in Alu regions, those in repetitive non-Alu regions and those in non-repetitive regions. We performed the repeat region annotation using the RepeatMasker file downloaded from the UCSC Table Browser [23]. The percentages of all 12 editing types in each region were calculated separately. Specifically, the percentage of Ato-G editing was calculated based on the strand information of the uploaded data, which must be specified by users via the uploading website interface. For strandspecific data, only the A-to-G editing in the forward (+) strand and the T-to-C editing in the reverse (-) stand were regarded as A-to-G editing. For non-strand-specific data, all the A-to-G editing and the T-to-C editing were regarded as A-to-G editing. After the calculation, the results were represented as a bar chart (Fig. 1a).

\section{Genomic feature annotation}

MIRIA can annotate RNA editing sites with a variety of useful genomic features. First, we used SnpEff [24], a genomic variant annotations tool, to annotate all RNA editing sites in the uploaded data. The annotation results were classified into six genomic clusters as follows: intergenic regions, intronic regions, CDS regions, ncRNAs (non-coding RNAs), 3'-UTRs and 5'-UTRs. Moreover, the corresponding gene name of each editing site was annotated. An overview annotation table could be accessed on the MIRIA web interface (Fig. 1b). Users could view all editing sites within one gene in an interactive visualization interface (Fig. 1c) by clicking on the gene name in the annotation table. Users could also explore the detailed information of the gene by clicking on the book icon adjacent to the gene name, which would directly link to the GeneCards page [25]. Besides the annotation table, MIRIA also generated an interactive bar chart to show the percentages of all the genomic features for each species (Fig. 1d).

\section{Overall editing level}

To examine the editing level statistics of each tissue in the uploaded data, we determined the editing level of each RNA editing site as the ratio of the number of reads supporting this site to the number of reads covering this site. The overall editing level statistics of each tissue were displayed using a boxplot (Fig. 1e).

\section{Genome-wide distribution of RNA editing sites}

For the statistical analysis of RNA editing sites on a genome-wide level, each chromosome was partitioned into contiguous $1-\mathrm{Mb}$ windows, and the total number of RNA editing sites was calculated within each window. Thereafter, an interactive Circos graph was generated to compare the different RNA editing numbers between the different tissues on a genome-wide level (Fig. 1f).

Table 1 The format of a single RNA editing file

\begin{tabular}{|c|c|c|c|c|c|c|}
\hline Chromosome & Location (0 base) & Location (1 base) & Type & Supporting information & Strand & AD:DP \\
\hline chr1 & $1,266,631$ & $1,266,632$ & $\mathrm{TC}$ & 3 & - & $5: 9$ \\
\hline chr1 & $1,280,297$ & $1,280,298$ & $A G$ & 2 & + & $2: 2$ \\
\hline
\end{tabular}

Chromosome: Name of chromosome. Location (0/1 base): Position of the RNA editing site in the chromosome. Type: Type of the identified RNA editing site (e.g., AG means 'A-to-G', etc.). Supporting information: Number of supporting reads for this RNA editing site. Strand: Possible values are: '.', ' + ', and ' - ,' which corresponded to 'uncertain', 'positive strand', and 'negative strand', respectively. AD:DP: Number of reads that had this RNA editing site, and the number of reads that covered this location 
a

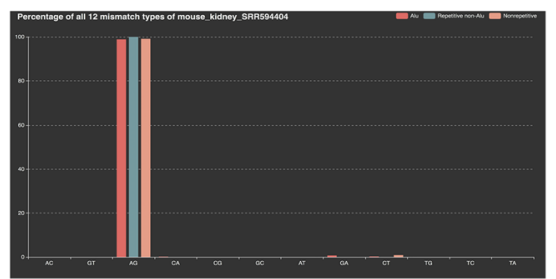

C

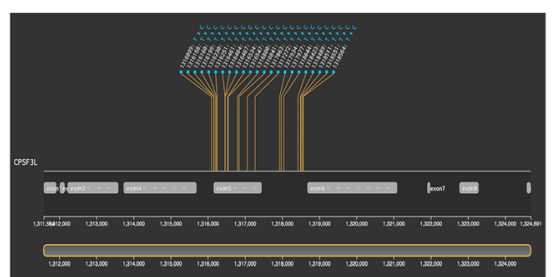

e

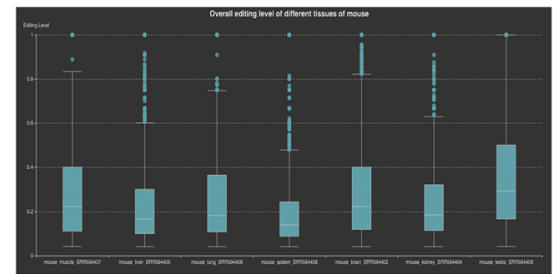

g

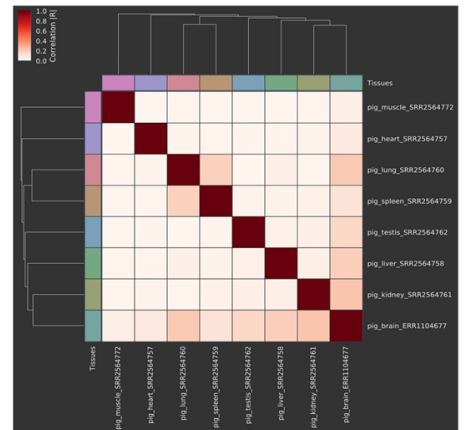

b

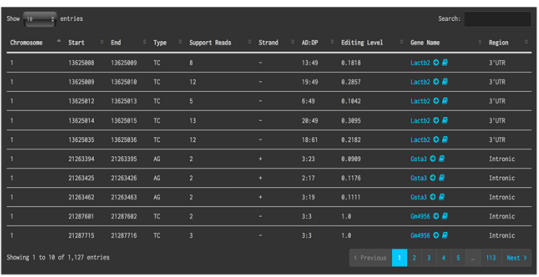

d

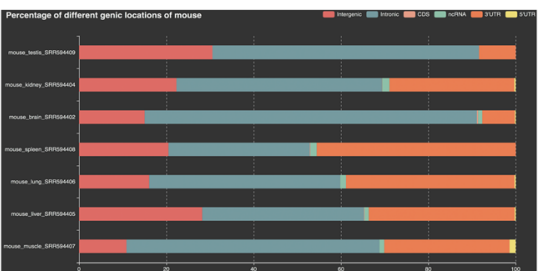

f

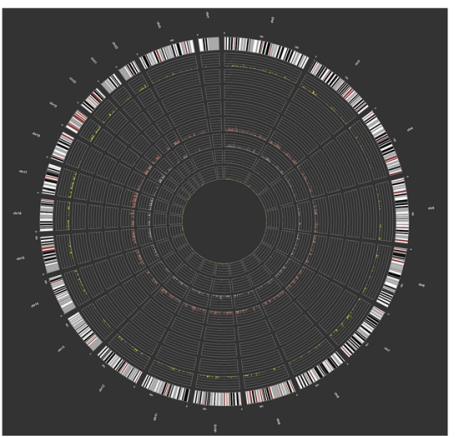

h

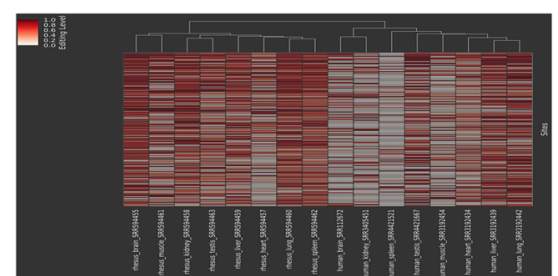

Fig. 1 Example outputs of MIRIA. a Percentages of all 12 RNA editing types in Alu regions, repetitive non-Alu regions and non-repetitive regions. b Overview annotation table for a tissue. c Visualization interface showing all RNA editing sites within a gene. $\mathbf{d}$ Percentages of different genomic features across tissues in a species. e The overall RNA editing level across tissues in a species. $\mathbf{f}$ A Circos graph comparing the difference in the RNA editing numbers between different tissues at the genome-wide level. $\mathbf{g}$ Pearson correlations for the RNA editing levels of editing sites between various tissues in a species. $\mathbf{h}$ Heatmap showing the RNA editing levels of the top 200 conserved sites for human and other mammalian tissues. The hierarchical clustering dendrogram of tissues based on the correlations of the editing levels between tissues was appended at the top of the heatmap

\section{Tissue-specific RNA editing}

To markedly improve the identification accuracy of the tissue-specific RNA editing sites, we removed the sites with a coverage less than 20 . We then merged the RNA editing sites of all tissues in one species to one matrix and designated the RNA editing sites as rows and the tissues as columns. The ROKU R package [26] was applied to rank the RNA editing sites by their tissue specificity using the Shannon entropy. All sites satisfying two requirements, namely, that the editing level range (i.e., maximum editing level minus the minimum editing level) was larger than 0.1 and the Shannon entropy was less than 0.4 were reserved as tissue-specific RNA editing sites. The Shannon entropy cutoff could be adjusted by users on the data uploading interface. The absolute value of the Pearson correlation for the RNA editing levels of the tissue-specific sites between tissues was presented as a heatmap (Fig. 1g).

\section{Conserved RNA editing}

To identify the conserved RNA editing sites in humans and other mammals, we adopted the UCSC LiftOver tool [23] to convert the genome position of each human reference to a mammalian reference. We also converted the genome position of other mammalian references to a human reference. The chain files of the human to the other mammalian references or the other mammals to the human reference were obtained from the UCSC 
download page. The RNA editing sites successfully converted on both turns (i.e., from the human to the other mammals and from the other mammals to the human) were reserved as conserved RNA editing sites. We used a heatmap to show the editing levels of the conserved RNA sites for tissues between the human and the other mammals. Moreover, the hierarchical clustering dendrogram of tissues based on the correlations of the RNA editing levels between tissues was appended at the top of the heatmap (Fig. 1h). By default, the heatmap only displayed the top 200 conserved RNA editing sites, which were sorted by the average editing level. Users had the option of adjusting the number of sites displayed in the heatmap on the data uploading interface.

\section{Results availability}

After the submission of an analysis request, MIRIA returned a job ID to users, and users could check their job status with the ID. After the job completion, users could view the results by clicking on the "view result" link on the check job status page. All the results provided by MIRIA are publication ready. For the Circos graph, the PNG or SVG image file could be downloaded by clicking on the download button at the top of the results page. For the other graphs, the PDF or SVG image files are available. Moreover, the annotation table for each sample could also be downloaded as a tab delimited text file (.tsv) from the overview page of the job results interface.

\section{Webserver implementation}

The MIRIA website was built using the Django Python Web framework [27] coupled with the MySQL database. The front-end interface was developed based on the Bootstrap open source toolkit [28]. The server-side data processing was supported by Docker [29]. The web interactive visualization graphs were developed using D3.js [30] and the ECharts [31] visualization library. The downloadable Circos graph was generated by the Circos software package, and the other graphs were produced using the ggplot $2 \mathrm{R}$ package and the Seaborn Python visualization library. MIRIA was published using the Apache Http server. The MIRIA website is freely available to all users, and there is no login requirement for accessing any of its features.

\section{Results}

To evaluate the MIRIA webserver, we collected 55 RNA-seq datasets from seven mammals (i.e., human, mouse, rat, rhesus, pig, cow, sheep) as the test data. For each mammal, seven or eight tissue samples (i.e., brain, liver, lung, kidney, spleen, testis, heart, muscle) were used. The RNA-seq data were downloaded from the National Center for Biotechnology Information (NCBI)
SRA database. The genomes and annotation files of the mammals were downloaded from the ENSEMBL database [32]. RepeatMasker files were downloaded from the UCSC table browser [23]. The reads were individually mapped to reference genomes using Hisat2 (v2.0.1) [33] with default parameters. The reference genomes used were as follows: human (hg38), mouse (mm10), rat (rn6), rhesus (rheMac8), pig (susScr3), cow (bosTau8) and sheep (oviAri3). The SAM files were sorted and converted to BAM files by Samtools (v1.2) [34] with default parameters. The RNA editing sites were identified using the "sprint_from_bam" program within SPRINT [35] with default parameters. We used the RNA editing sites data produced by SPRINT as the uploaded data for our webserver. The example results can be accessed by following the link https://mammal.deepomics.org/demo/.

\section{Conclusion}

We developed the MIRIA to provide both visualization and analysis of mammal RNA editing data. MIRIA enables experimental biologists without any computational programming skills to perform a diverse range of analyses including RNA editing type statistics, genomic feature annotations, editing level statistics, genome-wide distribution of RNA editing sites, tissue-specific analysis and conservation analysis. For every analysis, the result is presented with a visualized graph and can be downloaded as a publication-ready format. In general, with the functions of the MIRIA designed for mammal RNA editing data, we believe that this webserver will be a valuable resource for experimental biologists who are interested in revealing the functions of RNA editing sites.

\section{Availability and requirements \\ Project name: MIRIA \\ Project home page: https://mammal.deepomics.org/ \\ Operating system(s): Platform independent \\ Programming language: Python \\ Other requirements: Chrome, Safari, Firefox or IE \\ License: GNU GPL}

Any restrictions to use by non-academics: None

\section{Supplementary information}

Supplementary information accompanies this paper at https://doi.org/10. 1186/s12859-019-3242-2.

Additional file 1: Table S1. The data source from NCBI.

Abbreviations

ADAR: Adenosine Deaminase that Acts on RNA; CDS: Coding DNA Sequence; ncRNAs: non-coding RNAs; SRA: Sequence Read Archive; UTR: Untranslated Region

Acknowledgments

We thank Xiang Ao and Zhou Fang for their helpful suggestions and feedback. 


\section{About this supplement}

This article has been published as part of BMC Bioinformatics Volume 20 Supplement 24, 2019: The International Conference on Intelligent Biology and Medicine (ICIBM) 2019. The full contents of the supplement are available online at https://bmcbioinformatics.biomedcentral.com/articles/supplements/ volume-20-supplement-24.

\section{Authors' contributions}

SL, XF and ZW conceived and designed the webserver. XF and ZW contributed to data analysis. ZW collected data. XF and HL developed the website. ZW, XF and SL contributed to manuscript writing. All authors read and agreed to the final manuscript.

\section{Funding}

Publication costs are funded by a GRF Project grant from the RGC General Research Fund (9042181; CityU 11203115) and the GRF Research Project (9042348; CityU 11257316)

\section{Availability of data and materials}

All the data used in our research were downloaded from the National Center for Biotechnology Information (https://www.ncbi.nlm.nih.gov/). And the Sequence Read Archive (SRA) id can be found at Additional file 1: Table S1.

\section{Ethics approval and consent to participate}

Not applicable.

\section{Consent for publication}

Not applicable.

\section{Competing interests}

The authors declare that they have no competing interests.

\section{Published: 20 December 2019}

\section{References}

1. Bass BL. RNA editing by adenosine deaminases that act on RNA. Annu Rev Biochem. 2002;71(1):817-46.

2. Nishikura K. Functions and regulation of RNA editing by ADAR deaminases Annu Rev Biochem. 2010;79:321-49.

3. Gott JM, Emeson RB. Functions and mechanisms of RNA editing. Annu Rev Genet. 2000;34(1):499-531.

4. Lee J-H, Ang JK, Xiao X. Analysis and design of RNA sequencing experiments for identifying RNA editing and other single-nucleotide variants. RNA 2013:19(6):725-32.

5. Sommer B, Köhler M, Sprengel R, Seeburg PH. RNA editing in brain controls a determinant of ion flow in glutamate-gated channels. Cell. 1991;67(1):11-9.

6. Köhler M, Burnashev N, Sakmann B, Seeburg PH. Determinants of Ca2+ permeability in both TM1 and TM2 of high affinity kainate receptor channels: diversity by RNA editing. Neuron. 1993;10(3):491-500.

7. Lomeli H, Mosbacher J, Melcher T, Hoger T, Kuner T, Monyer H, Higuchi M, Bach A, Seeburg PH. Control of kinetic properties of AMPA receptor channels by nuclear RNA editing. Science. 1994:266(5191):1709-13.

8. Burns CM, Chu H, Rueter SM, Hutchinson LK, Canton H, Sanders-Bush E, Emeson RB. Regulation of serotonin-2C receptor G-protein coupling by RNA editing. Nature. 1997;387(6630):303

9. Casey JL, Gerin JL. Hepatitis D virus RNA editing: specific modification of adenosine in the antigenomic RNA. J Virol. 1995;69(12):7593-600.

10. Poison AG, Bass BL, Casey JL. RNA editing of hepatitis delta virus antigenome by dsRNA-adenosine deaminase. Nature. 1996;380(6573):454

11. Blow MJ, Grocock RJ, van Dongen S, Enright AJ, Dicks E, Futreal PA Wooster R, Stratton MR. RNA editing of human microRNAs. Genome Biol. 2006;7(4):R27.

12. Kume H, Hino K, Galipon J, Ui-Tei K. A-to-I editing in the miRNA seed region regulates target mRNA selection and silencing efficiency. Nucleic Acids Res. 2014:42(15):10050-60.

13. Zhang L, Yang C-S, Varelas X, Monti S. Altered RNA editing in 3' UTR perturbs microRNA-mediated regulation of oncogenes and tumorsuppressors. Sci Rep. 2016;6:23226.
14. Prasanth KV, Prasanth SG, Xuan Z, Hearn S, Freier SM, Bennett CF, Zhang $\mathrm{MQ}$, Spector DL. Regulating gene expression through RNA nuclear retention. Cell. 2005;123(2):249-63.

15. Rice Gl, Kasher PR, Forte GM, Mannion NM, Greenwood SM, Szynkiewicz M, Dickerson JE, Bhaskar SS, Zampini M, Briggs TA. Mutations in ADAR1 cause Aicardi-Goutieres syndrome associated with a type I interferon signature. Nat Genet. 2012:44(11):1243.

16. Toth AM, Li Z, Cattaneo R, Samuel CE. RNA-specific adenosine deaminase ADAR1 suppresses measles virus-induced apoptosis and activation of protein kinase PKR. J Biol Chem. 2009;284(43):29350-6.

17. Han L, Diao L, Yu S, Xu X, Li J, Zhang R, Yang Y, Werner HM, Eterovic AK, Yuan $Y$. The genomic landscape and clinical relevance of A-to-I RNA editing in human cancers. Cancer Cell. 2015:28(4):515-28.

18. Neeman $Y$, Levanon $E Y$, Jantsch MF, Eisenberg E. RNA editing level in the mouse is determined by the genomic repeat repertoire. RNA. 2006:12(10): 1802-9.

19. Picardi E, Regina TMR, Brennicke A, Quagliariello C. REDIdb: the RNA editing database. Nucleic Acids Res. 2006;35(suppl_1):D173-7.

20. Kiran A, Baranov PV. DARNED: a DAtabase of RNa EDiting in humans. Bioinformatics. 2010;26(14):1772-6.

21. Ramaswami G, Li JB. RADAR: a rigorously annotated database of A-to-I RNA editing. Nucleic Acids Res. 2013:42(D1):D109-13.

22. Picardi E, D'Erchia AM, Lo Giudice C, Pesole G. REDIportal: a comprehensive database of A-to-I RNA editing events in humans. Nucleic Acids Res. 2016; 45(D1):D750-7.

23. Meyer LR, Zweig AS, Hinrichs AS, Karolchik D, Kuhn RM, Wong M, Sloan CA Rosenbloom KR, Roe G, Rhead B. The UCSC genome browser database: extensions and updates 2013. Nucleic Acids Res. 2012:41(D1):D64-9.

24. Cingolani P, Platts A, Wang LL, Coon M, Nguyen T, Wang L, Land SJ, Lu X, Ruden DM. A program for annotating and predicting the effects of single nucleotide polymorphisms, SnpEff: SNPs in the genome of Drosophila melanogaster strain w1118; iso-2; iso-3. Fly. 2012;6(2):80-92

25. Safran M, Dalah I, Alexander J, Rosen N, Iny Stein T, Shmoish M, Nativ N, Bahir I, Doniger T, Krug H. GeneCards version 3: the human gene integrator. Database. 2010:2010:1-16.

26. Kadota K, Ye J, Nakai Y, Terada T, Shimizu K. ROKU: a novel method for identification of tissue-specific genes. BMC Bioinformatics. 2006:7(1):294.

27. Django: The Web framework for perfectionists with deadlines. https://www. djangoproject.com/. Accessed 23 Sept 2017.

28. Bootstrap - The most popular HTML, CSS, and JS library in the world. https:// getbootstrap.com/. Accessed 23 Sept 2017.

29. Docker - Build, Ship, and Run Any App, Anywhere. https://www.docker. com/. Accessed 19 Oct 2017.

30. D3.js - Data-Driven Documents. https://d3js.org/. Accessed 27 Oct 2017.

31. Echarts. https://ecomfe.github.io/echarts-doc/public/en/index.html. Accessed 14 Dec 2017.

32. Aken BL, Achuthan P, Akanni W, Amode MR, Bernsdorff F, Bhai J, Billis K Carvalho-Silva D, Cummins C, Clapham P. Ensembl 2017. Nucleic Acids Res. 2016;45(D1):D635-42.

33. Kim D, Langmead B, Salzberg SL. HISAT: a fast spliced aligner with low memory requirements. Nat Methods. 2015;12(4):357-60.

34. Li H. A statistical framework for SNP calling, mutation discovery, association mapping and population genetical parameter estimation from sequencing data. Bioinformatics. 2011;27(21):2987-93.

35. Zhang F, Lu Y, Yan S, Xing Q, Tian W. SPRINT: an SNP-free toolkit for identifying RNA editing sites. Bioinformatics (Oxford, England). 2017;33(22): 3538-48.

\section{Publisher's Note}

Springer Nature remains neutral with regard to jurisdictional claims in published maps and institutional affiliations. 